\title{
Development of Route Improvement Mechanisms for NDN
}

\author{
Vithayasard Yathortou ${ }^{1}$, Miaodi $^{2}$, Wang Jiuzhou ${ }^{3}$, Trat Siriyothin ${ }^{4}$, Sijingcheng ${ }^{5}$ \\ School of Electronic Engineering, Tianjin University of Technology and Education,
}

Tianjin, China 300222

\begin{abstract}
Named Data Networking (NDN) was developed to improve the network architecture. To be suitable for the current network connection type, which is Content-Distribution Which has a data packet named with a prefix and the recipients of the data use the interest packet sent to the network to find the desired data without worrying about the source and can Cache the data on the router device. Although the NDN network provides a way to make data available the name prefix is directed and can be backed up, but the NDN network architecture There are still many problems, and one of the important problems is Connection path improvements That needs to be Responsiveness that is not yet fast for changing of the network. In this research we designed and developed the Longest Prefix Reduction (LPR) for NDN networks by improving the routing cost of the connection path. To respond to failed connection routes faster, from this research helps improve the route faster, significantly helping Increase the transmission rate, get more information. Which allows connection in NDN network has improved performance.
\end{abstract}

Keywords-NDN, NFD, Route improvement, NDN Testbed.

\section{INTRODUCTION}

At present, the communication in the network will use the protocol TCP/IP [1] which has a connection pattern Point-toPoint and using only the TCP/IP protocol will not be effective when there are many services us ers, thus enabling the distribution of content (Content) to various sources, and must be costly and there are still issues of security issues Liang et al said [2]. In response to the above problems, research scholars have proposed a content-centric future network [2,3]. The Named Data Networking (NDN) was developed to improve the network architecture, to suit the current network connection. The Named Data Networking (NDN) [3] was developed to improve the network architecture to suit the current network connection. therefore, in this research designed and developed the improvement system path and selection of Longest Prefix Reduction (LPR) for the NDN network with improved Routing Cost of the connection path, to respond to failed connection path faster to improve the route improvement process for the NDN network, and Developing an extension of NFD software for selecting routes for forwarding packets in the NDN network.

Software that used for prototype development from the concept of This research has many software groups that support packet forwarding. In the NDN network, such as Named Forwarding Daemon (NFD) [4] and Content Centric Network (CCN) [5], which research has chosen NFD as an extension tool, because NFD is open source software and a prototype from a group of researchers NDN network and then test the prototype software on the system Testbed network.
From the results, it was found that the NFD from the work this research helps improve the route faster, significantly helping Increase the transmission rate, get more information. Which allows connection in NDN network has improved performance.

\section{BACKGROUND}

\subsection{Named Forwarding Daemon Software (NFD)}

NDN Forwarding Daemon (NFD) is a network forwarder that implements and evolves together with the Named Data Networking (NDN) protocol [16]. The main design goal of NFD is to support diverse experimentation with NDN architecture. NFD is developed as a tool for processing Interest and Data packets on the router device in the NDN network. The NFD has a structure as show in figure 3 . Acts as a multiplexer between routers consists of forwarder to manage the packet forwarding plan by using the Strategies module, and being the interface between the Face system, CS system, PIT and FIB etc. in the part of the Face System, it will manage the multiplex method which is currently able to work on Ethernet networks and overlay on TCP and UDP. When forwarder receives the prefix of the packets that will be sending to the destination, Forwarder will send the packet containing the prefix to the Face system and sent to the destination via various channels. In Forwarder it still connects to the CS, PIT and FIB systems according to the NDN network architecture mentioned above, which is managed using the NDN-Cxx library and is an important library for the NFD to process Interest and Data packets.

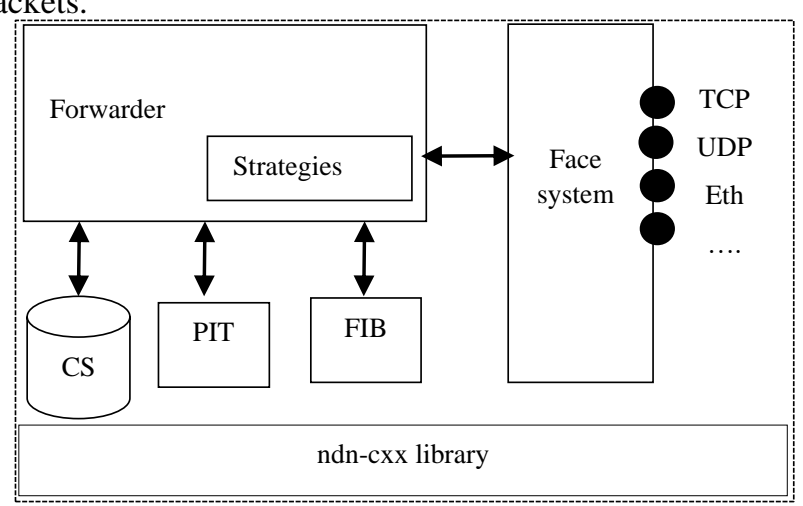

Figure 3 NFD Components

\subsection{Forwarding packets in the NDN network}

In the NDN network, there are many software developments, such as CCN [5], NFD [4] and development as an operating system Such as RIOS, etc. Every software has a working mechanism the mechanism in Figure 2, which the NDN network contains 2 types of packets: Interest and Data. In interest Packets do the duty to search for the desired data and the router has a working procedure as the following: 
1) When receiving an interest packet, the router will search for data packets that were previously backed up in Content Store (CS) and if found in CS, they will reply to the sender and end the work.

2) If the data cannot be found in CS, the system will bring the interest to check in the Pending Interest Table (PIT), which is a table to check the situation sending Interest under the same data before, if the interest has been previously exported the router will store the Face (Network Interface in the TCP / IP network architecture) to be used to send Data packets back through various faces with request, Therefore it's making the router does not send the Interest Packets On the redundant Upstream path.

3) In the case of an interest packet being checked in PIT and did not find the previous Interest submission. The system will check the Forwarding Information Base (FIB) table .By searching for a Face that will send the Interest to the destination with Longest Prefix Match algorithm (LPM) [8] and forward the packet from the router to find the next data, but if cannot use LPM to find a Face that will forward the packets. The router will notify the Interest sender on the Downstream by using the Negative ACK (NACK) packets to notify the previous router, there is no path to connect to the destination.

When the Router has received Data packet, the router will start checking the name of the data (Prefix). In the packet data type, if still in the PIT, it will forward the data packet via the Face list that was previously requested and make a backup (Cache) the data packet for using with the next request, but if cannot find the prefix of the data in the PIT table, The router will abandon (Drop) that data packets, because it is a strange data in the routing mechanism in the previous NDN network. [ 14,15].

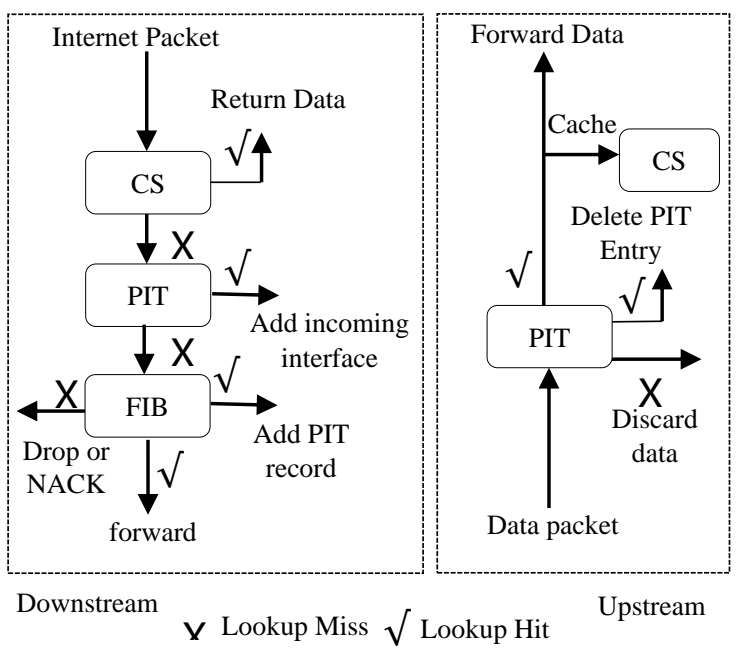

Figure 2 Packet processing and Forwarding by an NDN Router

The Algorithm that use to manage the path of sending the Interest Packets there are many algorithms such as: BR [8,9], NCC, Broadcast and Client. Control by BR is an algorithm that is used in the NFD for Forwarding a Interested Packet which BR works after receiving an interest packet and then using the Prefix of Interest check in PIT and if found prefix in PIT, the router will send the packet out to Face that has the lowest Routing Cost, but if cannot be found Packets, the packet will be taken to process with the Face that the last packet was sent not more than $100 \mathrm{~ms}$ and with the most similar prefix.

From using BR, you can see the Routing Cost is determined by using the Dijksra algorithm to calculate the distance to the destination in Multipath. According to the Named Link-State Routing protocol (NLSR) [10, 13], but setting the Routing Cost with that methods is permanently defined, making the using of the BR algorithm select to send packets via Face that cannot be connected Regardless of other factors such as: connection path failure etc. The BR algorithm is not effective enough to be used with various NDN networks, which it's the source of this research will design and develop in the route improvements automatic and up to date in network switching situations Software Named Forwarding Daemon.

\subsection{LPR designing and development}

Longest Prefix Reduction Algorithm (LPR) of this research there is a concept to improve the way of managing the Routing Cost together with managing the Prefix of Interest Packets. In selecting the connection route in the NDN network by adding the process of processing Interest Packets in the router as follows.

1)When the LPR receives an interest packet, it will select the Face that is used to forward the packet to Upstream from Face $\left(\mathrm{F}_{\text {out }}\right)$ with the lowest $\mathrm{C}$ and the longest prefix match by using the Longest Prefix Match algorithm that is in the NFD.

2)When $F_{\text {out }}$ takes Timeout, LPR will select the next Face with lowest $\mathrm{C}$ followed the faces until all faces are active.

3)If every Face cannot connect, LPR will reduce the Prefix of the Data name in the FIB table, it will decrease the prefix one by one and cannot connect. LPR will reduce the Prefix name in the Interest packet to / ndn / th / ac / tute / data and find the Face that supports sending new Interest as steps 1 and 2.

\subsection{Development and experiment of prototype programs.}

\subsubsection{Development of Assessment Interest Packet}

Prototype development based on the concept of LPR has been developed and integrated with NFD software by adding the function to process Interest Packets as shown in Figure 4 as follows:

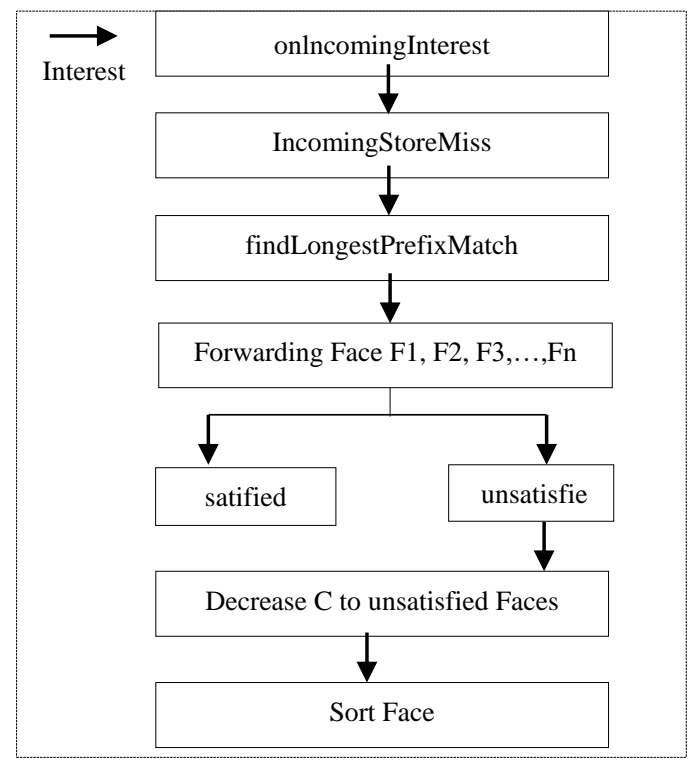

Figure 4 Interest Processing for LPR algorithm 
1)When the router receives an interest packet via Face at $\mathrm{F} 1, \mathrm{~F} 2, \mathrm{~F} 3, \ldots \mathrm{Fn}$, after De-multiplex the packet is sent to the function onIncomingIntest, which within onIncomingIntest will be checked according to the steps of the NDN architecture, but if not receiving data, the system will notify the function onContentStoreMiss then will enter to the route selection, Choose the Face to send the Interest Packets to the Upstream route.

2)Find Face that can send Interest Packets from the Face with Prefix that most matches Interest by the find longest prefix match function, after receiving the list of $F_{1}, F_{2}, F_{3}, \ldots$ $F_{n}$, that support sending packets, then select Face with the lowest Routing Cost $\left(\mathrm{F}_{\text {low }}\right)$ and send Interest Packets via such $\mathrm{F}_{\text {low. }}$.

3)When occur a Timeout via $F_{\text {low }}$ that has already sent an interest, NFD will report results to the Unsatisfied section and increase $\mathrm{C}$ one-by-one via $\mathrm{F}_{\text {low }}$ before rearranging all faces by sorting in ascending order and reducing the final prefix is decreased one-by-one according to the LPR algorithm, before sending a new Interest Packet (Retransmission) via the new Face with the lowest C, Development of Data Packets Processing.

When the router receives the data packet, it means that the face can be connected. Therefore, the Face with a high data transmission ratio from Downstream, there is a chance that the Interest transmission will receive high data, in the LPR algorithm there are improvement the Routing Cost (C) as steps in Figure 5.

When the router has received the data packet the system will be imported into the function onIncoming Data and then reduce $\mathrm{C}$ to the Face that received Data, then use the getNextHops function to get Face F1, F2, F3, ... Fi with the same prefix as the data that received before and reducing $\mathrm{C}$ from $\mathrm{C} 1, \mathrm{C} 2, \mathrm{C} 3, \ldots \mathrm{Ci}$ for every Face with the same Prefix to nourish the distance of $\mathrm{C}$ In each face, there is no difference too high and does not make the $\mathrm{C}$ higher without ending from the process Manage $\mathrm{C}$ in the processing of Interest Packets.

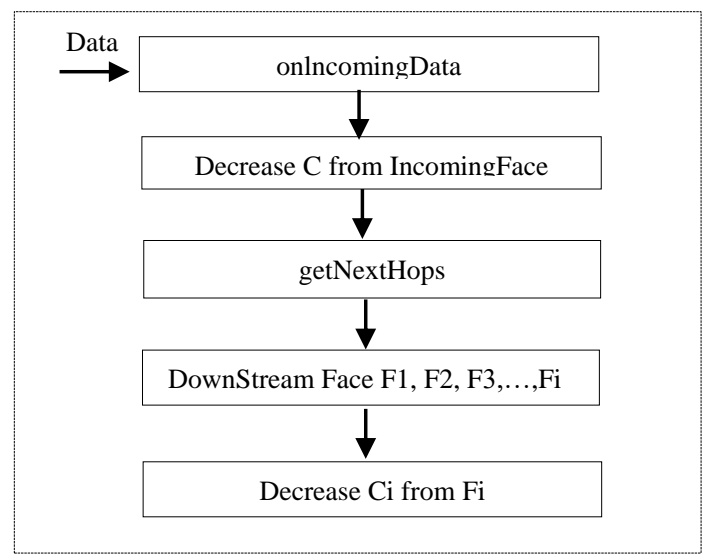

Figure 5 Data processing for LPR algorithm

III. EXPERIMENT METHOD

This experiment installs the NFD version 0.4.1 connected by using the internet hack. 192.168.6.0/24 and use all UDP faces and use VirtualBox software to create a Virtual Machine (VM), installing the Linux operating system, CentOS version 7 has a 1-core processor, 1024Gb RAM, 20Gb hard disk and a Bridge network configuration.
The Consumer machine is configured to connect to the NDN Router via UDP Face, by setting the Prefix / ndn for the FIB, this will cause every packet to be transferred to the NDN Router, at the same time the NDN Router uses UDP Face to connect to the NDN Testbed system.

The Consumer machine uses ndnping software to test to connect to the various nodes, according to the Prefix in Table1, and then evaluate the Convergence Time and Lost Rate of using the BR and LPR algorithms, before taking to analyze the results, in this research Requiring evaluation of efficiency and effectiveness using the range 95\% confidence, As figure 6 show an experiment diagram for testing BR and LPR algorithms.

Each Prefix from Table 1 to test Convergence Time, each node is sent a packet of 30 times for checking the Loss Rate, the Connection to the destination will be used 3 different times, 10,50 and 100 times Respectively, to observe the efficiency of resource of the test machine, and each time of packet transmission will control the parameters at the local network level, it's containing 100Mbps bandwidth channel, and not allowing external packets to interfere with the testbed system In the local network of this research.

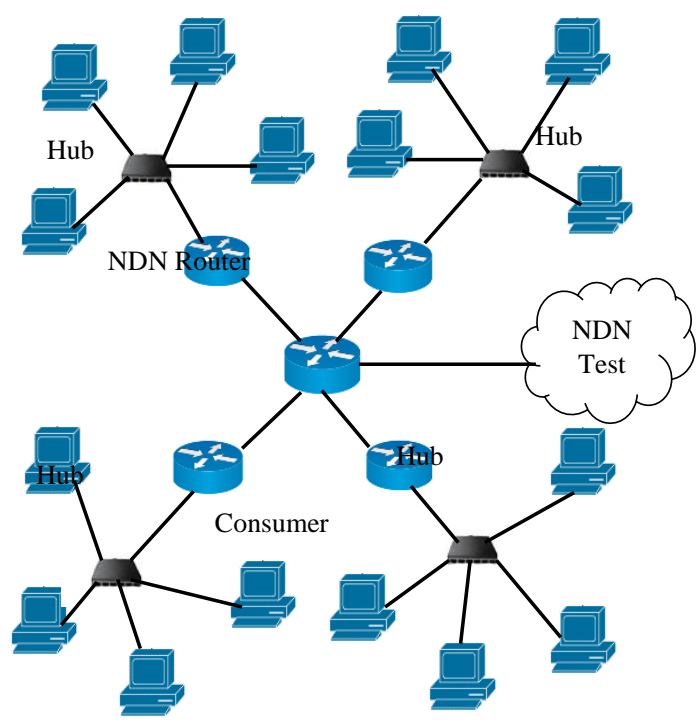

Figure 6 an evaluation scenario

Table 1 Testbed Prefixes

\begin{tabular}{|c|c|}
\hline /ndn/br/ufpa & /ndn/ch/unibas \\
\hline /ndn/de/uni-goettingen & /ndn/edu/Arizona \\
\hline /ndn/edu/byu & $/$ ndn/edu/colostate \\
\hline /ndn/edu/Illinois & /ndn/edu/Memphis \\
\hline /ndn/edu/neu & $/$ ndn/edu/uci \\
\hline /ndn/edu/ucla & $/$ ndn/edu/ucla/remap \\
\hline /ndn/edu/wustl & $/$ ndn/es/urjc \\
\hline /ndn/fr/unip6 & $/$ ndn/gov/nist \\
\hline /ndn/it/unipd & $/$ ndn/jp/ac/osaka-u \\
\hline /ndn/anyang & $/$ ndn/kr/re/kisti \\
\hline /ndn/no/ntnu & $/$ ndn/pt/ulusofona/copelabs \\
\hline$/$ ndn/pt/uminho & $/$ ndn/cn/edu/tongji \\
\hline /ndn/id/ac/ui & $/$ ndn/nl/tno \\
\hline /ndn/org/caida & $/$ ndn/com/orange \\
\hline /ndn/edu/umich & $/$ ndn/biz/xp3/yayatute \\
\hline
\end{tabular}




\section{NFD ASSESSMENT RESULTS}

\subsection{Convergence Time Assessment Results}

In evaluating the Convergence Time between the use of BR and LPR algorithms. The experimental results show the efficiency of the LPR, that help the Convergence Time to be lower than using BR, which results in Figure 8 being the Convergence Time of the LPR algorithm. In the X-axis is the test node that has all 30 nodes, and the $\mathrm{Y}$-axis is the Convergence Time that occurs after the route is updated in milliseconds. This research uses the average value obtained from the ndnping and LPR software. The overall convergence time is between $5095.5 \pm 9.04 \mathrm{~ms}$ is different from using BR, which cannot connect to the destination, therefore Timeout occurred and there was no result in any path improvement.

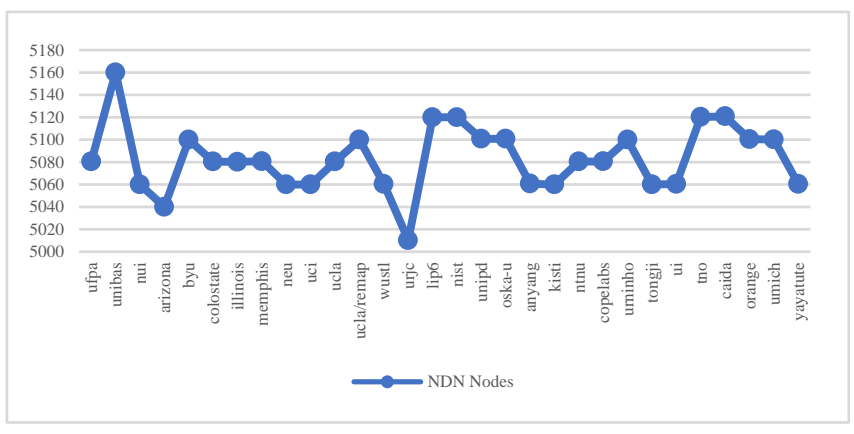

Figure 8 LPR Convergence Time

\subsection{Loss Rate Assessment Results}

Loss Rate is an important factor to make the efficiency and the effectiveness of the transmission of packets in high volumes, The result of the evaluation between the use of the $\mathrm{BR}$ and LPR algorithms is shown in Figure 9 in the X-axis is the number of packets sent to the destination and the $\mathrm{Y}$-axis is the percentage of loss rate. From the results of the experiment, it was found that the use of BR algorithms, it's made 100 percent packet loss, because of the response to the connection is delayed and the BR algorithm does not choose a new route until it receives NACK packets and deletes the route from the FIB, but in the experiment on the testbed system in this research 1) The NACK packets are not returned to the Consumer, because the NDN Router has FIB tables and PIT tables that wait for Timeout and be updated with Interest Packets continuously.

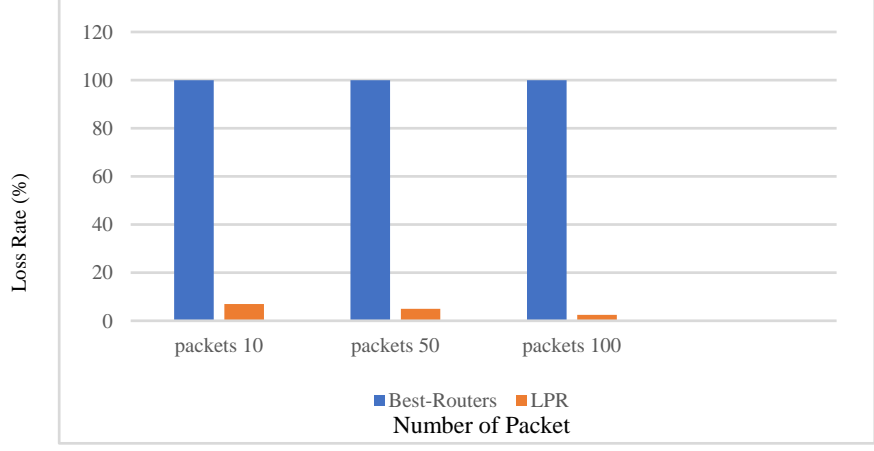

Figure 9 Loss Rate of Best-Route and LPR

2) Cost adjustment of the BR algorithm is customized, by the administrator, which make BR must choose the same route at all times, Even if unable to connect, Causing the transmission of packets of 10,50 and 100 to Testbed cannot be made, but when switching to the LPR algorithm, it is seen that when sending packets of 10 packets, there will be a loss rate of $2.08 \pm 4.0$ percent, when increasing the number of packets to 50 , Loss Rate packets has a ratio reduce to $1.67 \pm 1.78$ percent, and in the case of sending 100 packets of packet the Loss Rate is reduced to $0.9 \pm 1.10$ percent, from the results of this experiment show the LPR algorithms is able to adjust the Routing Cost and allow the NFD to choose the route to send the Interest Pack via Face that can be used quickly and help increase both the efficiency and effectiveness of communication in the NDN network.

\subsection{Performance evaluation}

Assessment of the use of forecasting from this research, CPU and RAM usage was tested by collecting data every 5 minutes during the experiment, 30 times. Found that the rate of managing memory in figure 10 can be seen that the LPR algorithm has a higher memory utilization rate than the BR algorithm, which is due to the addition of the Routing Cost function and reducing the Prefix to find Routes in the NDN network, but the use of the LPR memory algorithm is relatively stable and slightly higher than the BR algorithm.

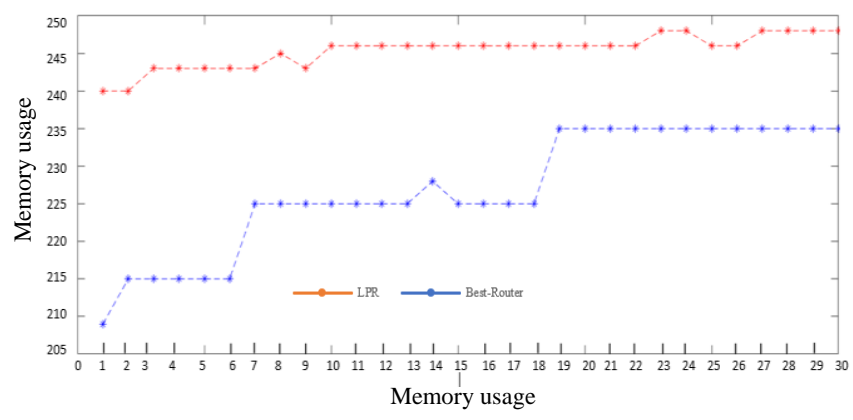

Figure 10 Memory Load

\section{CONCLUSION}

The NDN network architecture was built to suit to apply in the era that content is important for applications in the NDN network, Named Prefix is used as a tool for receiving content using interest and data packets. Using Named Prefix as a packet stimulation tool allows NDN to connect to the multipath network. Currently, NDN uses the BR algorithm and makes decisions using Routing Cost. There are still many problems such as respond to route selection, connection failed, delayed. In this research therefore developed the LPR algorithm to help improve the route of choosing packet delivery routes to support multipath packet delivery by managing the method of sending interest packets by improving the flexible routing cost method.

This research introduced the development concept in NFD software tested on NDN Testbed system and test results. Shows the efficiency and effectiveness of the route improvement of interest packets, which helps the LPR to have a low rate of $90 \%$ that the BR usage is more than $90 \%$.

\section{REFERENCE}

Wollschlaeger, Martin, Thilo Sauter, and Juergen Jasperneite. "The future of industrial communication: Automation networks in the era of the internet of things and industry 4.0." IEEE industrial electronics magazine 11, no. 1 (2017): 17-27.

[2] Liang, Jinjin, Jian Jiang, Haixin Duan, Kang Li, Tao Wan, and Jianping Wu. "When HTTPS meets CDN: A case of authentication in 
delegated service." In 2014 IEEE Symposium on Security and Privacy, pp. 67-82. IEEE, 2014

[3] Zhang, Lixia, Alexander Afanasyev, Jeffrey Burke, Van Jacobson, Patrick Crowley, Christos Papadopoulos, Lan Wang, and Beichuan Zhang. "Named data networking." ACM SIGCOMM Computer Communication Review 44, no. 3 (2014): 66-73.

[4] Yu, Yingdi. "Public key management in named data networking." NDN, Univ. California, Los Angeles, CA, USA, Tech. Rep. NDN-0029 (2015).

[5] Westphal, Cedric. "Method for network coding packets in contentcentric networking based networks." U.S. Patent 9,002,921, issued April 7, 2015.

[6] Marxer, Claudio, Christopher Scherb, and Christian Tschudin. "Access-controlled in-network processing of named data." In Proceedings of the 3rd ACM Conference on Information-Centric Networking, pp. 77-82. ACM, 2016.

[7] Zhang, Lixia, Alexander Afanasyev, Jeffrey Burke, Van Jacobson, Patrick Crowley, Christos Papadopoulos, Lan Wang, and Beichuan Zhang. "Named data networking." ACM SIGCOMM Computer Communication Review 44, no. 3 (2014): 66-73. [8] Afanasyev et al A. NFD Developer's Guide. University of California, Los Angeles; 2014 Aug. Report No.: NDN-0021. p. 1-60.

[9] Phyo, K-zin, and Myint Myint Sein. "Effective Evacuation Route Strategy during Natural Disaster." Proceedings of the Asia-Pacific Advanced Network 44 (2017): 70-75.

[10] Hoque, A. K. M., Syed Obaid Amin, Adam Alyyan, Beichuan Zhang, Lixia Zhang, and Lan Wang. "NLSR: named-data link state routing protocol." In Proceedings of the 3rd ACM SIGCOMM workshop on Information-centric networking, pp. 15-20. ACM, 2013.

[11] Yu, Yingdi, Alexander Afanasyev, David Clark, Van Jacobson, and Lixia Zhang. "Schematizing trust in named data networking." In Proceedings of the 2nd ACM Conference on Information-Centric Networking, pp. 177-186. ACM, 2015.

[12] Berman, Mark, Piet Demeester, Jae Woo Lee, Kiran Nagaraja, Michael Zink, Didier Colle, Dilip Kumar Krishnappa et al. "Future internets escape the simulator." Communications of the ACM 58, no. 6 (2015): 78-89.

[13] Shi, Junxiao, Eric Newberry, and Beichuan Zhang. "On broadcastbased self-learning in named data networking." In 2017 IFIP Networking Conference (IFIP Networking) and Workshops, pp. 1-9. IEEE, 2017.

[14] Yi, Cheng, Alexander Afanasyev, Ilya Moiseenko, Lan Wang, Beichuan Zhang, and Lixia Zhang. "A case for stateful forwarding plane." Computer Communications 36, no. 7 (2013): 779-791.

[15] Schneider, Klaus, Cheng Yi, Beichuan Zhang, and Lixia Zhang. "A practical congestion control scheme for named data networking." In Proceedings of the 3rd ACM Conference on Information-Centric Networking, pp. 21-30. ACM, 2016. 